https://helda.helsinki.fi

\title{
Eating practices and dietary health
}

\section{Holm, Lotte}

Bloomsbury Academic

2019

Holm , L , Lund , T B \& Niva , M 2019 , Eating practices and dietary health . in J Gronow \& L Holm (eds), Everyday eating in Denmark, Finland, Norway and Sweden : A comparative pÿstudy of meal patterns 19972012 . Bloomsbury Academic, London \& New York, pp. pÿ159 171 .

http://hdl.handle.net/10138/314429

acceptedVersion

Downloaded from Helda, University of Helsinki institutional repository.

This is an electronic reprint of the original article.

This reprint may differ from the original in pagination and typographic detail.

Please cite the original version. 
Holm L, Lund TB, Niva M (2019) Eating practices and dietary health. In: Everyday eating in Denmark, Finland, Norway and Sweden. A comparative study of meal patterns 1997-2012, ed. by J Gronow, L Holm, 159-171. London \& New York: Bloomsbury Academic. (Authors' accepted manuscript.)

\section{Eating practices and dietary health}

\section{Lotte Holm, Thomas Bøker Lund and Mari Niva}

What makes individuals eat healthily? The answer to this question is central for interventions and policies aiming to promote dietary health in populations. Social psychological approaches have shown that cognitive factors, such as reflexivity, and deliberate decisions and intentions promote dietary health (Bandura 2004), and that the motivation to eat healthily has an effect on intentions and behaviors related to healthy eating (Backman et al. 2002; Brug 2008; Connor et al. 2002; Sheeshka et al. 1993).

However, other approaches suggest that it is rather the daily practices around which eating revolves, practices embedded in the social and cultural contexts of daily life, that are the important factors influencing food intake(Connors et al. 2001; Gronow and Warde 2001; Halkier et al. 2011; Riet et al. 2011) and the healthiness of diets (van Woerkum and Bouwman 2014).

Adopting the latter perspective, this paper analyzes the relationship between dietary health and the following eating practices: social company while eating, the regularity and duration of eating, and activities while eating such as watching television.

Research into the way social company during eating influences food intake points in various directions(Herman et al. 2003;Sobal 2000). It has been shown that the mere presence of others will lead people to eat more, and even more so when more people are present(de Castro et al. 1990; de Castro and de Castro 1989), and there is some indication that the duration of eating episodes is the mediator of this social facilitation, as more time is spent eating when more people are present(Bell and Pliner 2003;Feunekes et al. 1995; Sommer and Steele 1997). Some studies suggest that meals eaten in company contain more carbohydrates, fat, protein and calories than meals eaten in solitude(de Castro, Brewer, Elmore, \& Orozco 1990; Redd and de Castro 1992). Others have found that people eat less healthily when eating alone(Donkin et al. 1998; Pliner and Bell 2009).

Television viewing is another daily habit which has been linked to dietary health. Eating while watching television is associated with higher energy intake(Granner and Mburia-Mwalili 2010; Jeffery and French 1998), with eating more high-density foods(Blass et al. 2006), and with greater intake of fat and sugar (Fuglestad et al. 2012) and lower intake of fruit and vegetables (Liang et al. 2009). It is also linked with poorer dietary quality when it takes place during family meals(Feldman et al. 2007), and in general with less healthy diets(Sisson et al. 2012).

Studies of meal patterns and dietary health point in several directions. Positive associations have been found between frequency of eating and intake of nutrients(Kerver et al. 2006) and healthiness of diets(Holmbäck et al. 2010). Negative associations have been identified between the irregular consumption of main meals, on the one hand, and fruit and vegetable intake (Fuglestad, Jeffery, \& 
Sherwood 2012; Lazzeri et al. 2013; Pedersen et al. 2012), nutrient intake(Affenito et al. 2005; Sjöberg et al. 2003) and the general healthiness of diets(Storey et al. 2009), on the other. While some studies find that frequent snacking is associated with high intake of sugared and fatty products, and low nutrient intake(Chaplin and Smith 2011; Ovaskainen et al. 2006), others conclude that the healthiness of snack-based diets does not differ from that in diets based more solidly on main meals,(Ovaskainen et al. 2010), and even that frequency of snacking is positively associated with more balanced and nutrient-dense diets(Whybrow and Kirk 1997; Zizza and Xu 2012).

The picture we have, then, is this: research appears to show both positive and negative associations between dietary health and eating practices such as social or solitary eating, meal frequency, regularity, and duration of meals; but where TV-viewing is concerned it identifies only negative associations. Most studies address at most one or a few of these activities, so little is known about the ways in which combinations of them impact on the healthiness of eating. Existing research includes studies based on small convenience samples(de Castro \& de Castro 1989; Pliner \& Bell 2009) and studies that are representative only of specific subpopulations (school children (Pedersen, Meilstrup, Holstein, \& Rasmussen 2012), adolescents (Sjöberg, Hallberg, Höglund, \& Hulthén 2003), women (Granner \& Mburia-Mwalili 2010) etc.) Further, how combinations of several eating practices affect dietary health at the national population level has not been examined, and it is unclear whether these effects (if there are such) might vary with cultural context, or whether they are essentially uniform. Nor is it clear whether the practices have a significant effect on dietary health when motivation to eat healthily is taken into account.

Against this background the present study uses population-level data from four Nordic countries (Denmark, Finland, Norway, and Sweden). For each country we analyze the association between dietary health and a number of eating-related activities, while controlling for healthy eating motivation. We identify sociologically relevant combinations of eating activities, which influence dietary health in each country and analyze their combined effect. The employment of data on populations in four countries makes it possible to investigate and discuss whether links between eating activities and dietary health vary with cultural context.

In the present analysis a sub-sample is used ( $\mathrm{N}=7531)$ owing to missing nformation on some of the measures employed. There are only minor variations in country-specific census data on gender, age, and household type. Where education was concerned there were more serious deviations from the population census in all countries, and in particular the population segment with the lowest level of education (individuals completing at most compulsory school or short-term specialization courses) was clearly under-represented. We therefore employed an education*gender*age weight variable in univariate analysis. In the present analysis a sub-sample is used $(\mathrm{N}=7531)$ owing to missing information on some of the measures employed.

The questionnaire invited respondents to register detailed accounts of one day of eating, in a manner similar to the 24-hour recall. All eating events (apart from minor episodes, such as chewing gum or pastilles) were included in the interview. The questionnaire focused on social aspects of eating events, such as eating alone/socially, the duration of events and activities during eating events. Further details of the questionnaire have been reported elsewhere (Lund and Gronow 2014). 
The dependent variable used in the analysis - Dietary Quality Score (DQS) - was adopted from Toft et al.(Toft et al. 2007). DQS is a validated and brief measure of the healthiness of diets focusing on four components: fats, vegetables, fruits and fish. It includes eight food frequency questions on the basis of which respondents are assigned a score (range: 1-9). Individuals were classified into three dietary groups: unhealthy (DQS SCORE 1-3), average (DQS $_{\text {SCORE }} 4-6$ ), and healthy (DQS $\left.S_{S C R E} 7-9\right)$. This measure was originally developed and validated with reference to eating habits in Denmark (Toft, Kristoffersen, Ladelund, Bysted, Jakobsen, Lau, Jorgensen, BorchJohnsen, \& Ovesen 2007). Therefore, the questions may not reflect the specific eating traits of Finns, Norwegians and Swedes as accurately as those of Danes. On the other hand, there are clear overlaps in eating culture in the four countries - indeed to such an extent that currently efforts are being made to construct a single tool to monitor diet in the four Nordic populations (Fagt et al. 2011). For this reason it is likely that the measure captures the same underlying behavior with respect to dietary quality.

Measures of eating-related activities were developed on the basis of the detailed recording of practical and contextual aspects of eating events during the 24 hours covered. They included meal regularity, where three variables were constructed: skipping breakfast, skipping lunch, and skipping dinner; frequency of meals, with three categories: 0-3 meals, 4 meals, 5 or more meals; proportion of short meals, constructed as the percentage of meals lasting 10 minutes or less, recoded into three gradient categories (low $=0-33.33 \%$, medium $=33.34-66.66 \%$, high $=66.67$ $100 \%$ ); proportion of TVmeals, constructed as the percentage of meals eaten while watching television, recoded likewise into the three gradient categories mentioned above; and proportion of meals eaten alone, constructed as the percentage of meals eaten alone, recoded into five gradient categories (very low $=0 \%$, low $=0.01-33.33 \%$, medium $=33.34-66.66 \%$, high $=66.67-99.99 \%$, very high $=100 \%$ ). With the exception of the meal regularity measure the term 'meals' here refers to all types of eating event.

A measure of motivation to eat healthily was developed using four questions (see Table 1 for details) with Likert-scale response options (indicating extent of agreement/disagreement). From this a composite variable was developed and rescaled to range from 0 (lowest motivation) to 100 (highest). The measure exhibited acceptable reliability in all countries (Cronbach's alpha = Denmark 0.65, Finland 0.65, Norway 0.63, and Sweden 0.59).

Standard socio-demographic measures were also included in the analysis (age, sex, household composition, educational level, occupational status, and the ESeC measure of social class(Rose et al. 2007)), to control for the possibility that socio-economic variables were confounding associations between eating activities and dietary health.

\section{Data analysis}

Ordered logistic regression analysis (using the ologit command in Stata 12) was run on a countryby-country basis with DQS inserted as the dependent variable, while the remaining variables described above were entered as independent variables. Effects of the measures of eatingrelated activities are displayed and discussed together with healthy eating motivation, while the sociodemographic variables are used as control variables (Table 10.2.). 
To evaluate the combined effect of several eating-related activities we set up two profiles with negative and positive effects on dietary quality, respectively (Table 10.3.). This was done by combining values of the country-specific eating activity variables that predicted dietary health significantly in the ordered logistic regression analysis. We aimed to construct profiles encompassing approximately $10-20 \%$ of the populations so as to ensure they were relatively substantial in size. For this reason not all of the statistically significant activity variables were used in the formulation of negative and positive profiles, since this would have entailed very infrequent profiles.

Changes in predicted probabilities (Table 10.4.) were calculated in order to interpret the effect of the independent variables on dietary quality. Predicted probabilities were calculated at three levels of motivation reflecting the gradient continuum of this factor $\left(10^{\text {th }}, 50^{\text {th }}\right.$, and $90^{\text {th }}$ percentile).

Changes in predicted probabilities caused by eating activities were computed for all statistically significant activity variables, and for the negative and positive eating activity profile. All predicted probabilities were calculated while holding the remaining variables at their mean value (using the margins command in Stata). 
Table 10.1: Descriptive statistics of measures of Dietary Quality Score, eating-related activities, and healthy eating motivation (column percentages or averages (s.d.))

$\begin{array}{cccc}\text { Denmark } & \text { Finland } & \text { Norway } & \text { Sweden } \\ (\mathrm{N}=1860) & (\mathrm{N}=1830) & (\mathrm{N}=1909) & (\mathrm{N}=1924)\end{array}$

\begin{tabular}{|c|c|c|c|c|c|}
\hline \multirow[t]{3}{*}{ Dietary quality } & Unhealthy & $22,7 \%$ & $27,3 \%$ & $21,3 \%$ & $25,8 \%$ \\
\hline & Average & $64,8 \%$ & $67,9 \%$ & $68,3 \%$ & $69,0 \%$ \\
\hline & Healthy & $12,5 \%$ & $4,9 \%$ & $10,4 \%$ & $5,1 \%$ \\
\hline \multirow[t]{3}{*}{ Meal regularity: skipping } & Breakfast & $11,2 \%$ & $14,3 \%$ & $12,7 \%$ & $7,5 \%$ \\
\hline & Lunch & $13,7 \%$ & $29,3 \%$ & $26,1 \%$ & $23,5 \%$ \\
\hline & Dinner & $5,1 \%$ & $28,3 \%$ & $9,1 \%$ & $24,0 \%$ \\
\hline \multirow[t]{4}{*}{ Frequency of meals } & $0-3$ meals & $43,0 \%$ & $39,4 \%$ & $42,9 \%$ & $45,5 \%$ \\
\hline & 4 meals & $26,3 \%$ & $33,1 \%$ & $34,7 \%$ & $31,8 \%$ \\
\hline & 5 or more & $30,7 \%$ & $27,6 \%$ & $22,4 \%$ & $22,8 \%$ \\
\hline & Low & $86,3 \%$ & $77,7 \%$ & $86,1 \%$ & $84,6 \%$ \\
\hline \multirow[t]{2}{*}{ Proportion of short meals } & Medium & $11,5 \%$ & $18,4 \%$ & $11,4 \%$ & $13,1 \%$ \\
\hline & High & $2,1 \%$ & $4,0 \%$ & $2,6 \%$ & $2,2 \%$ \\
\hline \multirow[t]{6}{*}{ Proportion of TV-meals ${ }^{1}$} & Low & $70,3 \%$ & $76,8 \%$ & $75,1 \%$ & $78,4 \%$ \\
\hline & Medium & $22,1 \%$ & $16,3 \%$ & $17,9 \%$ & $15,1 \%$ \\
\hline & High & $7,7 \%$ & $6,9 \%$ & $7,0 \%$ & $6,5 \%$ \\
\hline & Very low & $28,1 \%$ & $27,0 \%$ & $22,9 \%$ & $31,9 \%$ \\
\hline & Low & $25,7 \%$ & $22,0 \%$ & $23,4 \%$ & $25,9 \%$ \\
\hline & Medium & $18,3 \%$ & $17,8 \%$ & $26,0 \%$ & $16,8 \%$ \\
\hline \multirow[t]{2}{*}{ Proportion of meals eaten alone ${ }^{2}$} & High & $14,9 \%$ & $15,1 \%$ & $17,1 \%$ & $14,2 \%$ \\
\hline & Very high & $13,1 \%$ & $18,1 \%$ & $10,6 \%$ & $11,1 \%$ \\
\hline \multicolumn{2}{|c|}{ Avg. healthy eating motivation ${ }^{3}$} & 53,2 & 54,5 & 52,1 & 50,1 \\
\hline \multicolumn{2}{|l|}{ (s.d.) } & 18,3 & 18,1 & 18,0 & 18,5 \\
\hline
\end{tabular}

\footnotetext{
${ }^{1}$ Computed as percentage of meals during the day (Low $=0-33.33 \%$ of meals, Medium $=33.34-66.66 \%$, High $=66.67100 \%$ ).

${ }^{2}$ Computed as percentage of meals during the day (Very low $=$ no meals eaten alone, Low $=0.1-33.33 \%$ of meals, Medium $=33.34-66.66 \%$, High $=66.67-$ $99.99 \%$, Very high=All meals eaten alone)

${ }^{3}$ The following questions were used to construct the index: 1 : “To what degree did you think about the health or risks associated with the foods you ate yesterday? (five response options, indicating degree of health and risk concern)"; 2: "I am ready to hold back in my everyday diet if this gives me better health in the long run" (four response options indicating degree of agreement with statement); 3: "I am more concerned about the taste of food than how healthy it is" (four response options indicating degree of agreement with statement-item was reverse scored); , 4: "It's important to stick to the advice of experts on healthy eating" (four response options indicating degree of agreement with statement).
} 


\section{Results}

The descriptive results set out in Table 10.1. show that in all countries a small proportion of people skipped breakfast (ranging from 7.5\% in Sweden to 14.3\% in Finland). Approximately one in four skipped lunch and dinner in Finland (29.3\% and 28.3\%, respectively) and Sweden (23.5\% and $24.0 \%)$. In Denmark a small proportion skipped lunch (13.7\%) and dinner (5.1\%), while in Norway $26.1 \%$ skipped lunch and $9.1 \%$ skipped dinner. Three or fewer meals per day were most frequent (39.4\% in Finland to $45.5 \%$ in Sweden). Across all countries a large majority of participants ( $77.7 \%$ in Finland to $86.3 \%$ in Denmark) had only a limited proportion of short meals. Frequent TVwatching while eating was also relatively rare, as in all countries approximately three-quarters of participants (70.3\% in Denmark to $78.8 \%$ in Sweden) watched TV during only a small proportion of their daily meals. The extent to which meals were eaten alone was relatively evenly spread out across all categories from very high to very low, although the very low category (indicating that no meals were eaten alone) was most common (22.9\% in Norway to $31.9 \%$ in Sweden).

Results from the ordered logistic regression (Table 10.2.) show that healthy eating motivation predicts the dietary quality score positively in all countries. 
Table 10.2. Results from ordered logistic regression (dependent variable Dietary Quality)*

Denmark

Finland

Norway

Sweden

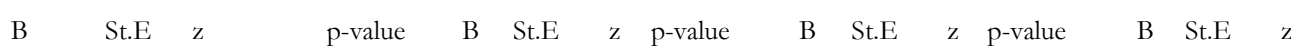

Skipped main meal

breakfast

$-0,296 \quad 0,185-1,600$

0,110

$-0,520 \quad 0,172-3,0200,003$

0,021

lunch

$-0,365^{0,159-2,300}$

dinner

0,985

$-0,286 \quad 0,134-2,1300,033$

$-0,020 \quad 0,128-0,1600,875$

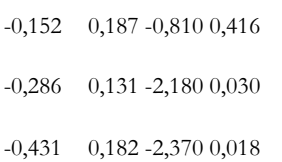

$0,070 \quad 0,2090,330$

0,738

$-0,345 \quad 0,132-2,620$

0,009

0,004

Proportion of meals

with short duration

(ref: high)

$$
\begin{array}{cc}
\text { Medium } & 0,1330,3760,350 \\
& 0,008 \\
\text { Low } & 0,3540,020
\end{array}
$$

0,724

$\begin{array}{lll}0,273 & 0,279 & 0,980\end{array}$

0,328

$-0,176 \quad 0,329-0,5400,592$

$-0,040 \quad 0,383-0,100$

0,917

0,982

$0,477 \quad 0,2641,810$

0,071

$-0,367 \quad 0,302-1,2100,225$

$0,141 \quad 0,3620,390$

0,697

Proportion of TV-meals

(ref: high)

$$
\begin{aligned}
& \text { medium } \\
& 0,237 \quad 0,2301,030
\end{aligned}
$$

0,304

$-0,235 \quad 0,261-0,9000,367$

$0,146 \quad 0,2450,590 \quad 0,553$

$0,358 \quad 0,2491,440$

0,151

0,030

$0,144 \quad 0,2380,600 \quad 0,546$

$0,100 \quad 0,2240,440 \quad 0,657$

$0,510 \quad 0,2222,300$

0,022

Proportion of meals eaten

alone (ref: very high)

$\begin{array}{ccc}\text { High } & 0,071 & 0,2040,350 \\ \text { medium } & 0,221 & 0,2081,060 \\ \text { low } & 0,590 & 0,2042,890 \\ \text { very low } & 0,578 & 0,2022,860\end{array}$

0,729

$0,372 \quad 0,199 \quad 1,870$

0,062

0,288

$0,201 \quad 0,2040,990$

0,325

$0,403 \quad 0,2141,880 \quad 0,060$

$0,011 \quad 0,2230,050$

0,961

0,004

$-0,103 \quad 0,204-0,5100,613$

$0,261 \quad 0,218 \quad 1,200 \quad 0,230$

$-0,023 \quad 0,232-0,100$

0,922

0,004

$0,010 \quad 0,2010,050 \quad 0,959$

$0,182 \quad 0,2160,840 \quad 0,400$

$0,068 \quad 0,2220,310$

0,758

0,431

Number of daily

meals (ref: $0-3$ )

$$
\begin{aligned}
& 4 \text { meals } \\
& 5 \text { or more } \\
& \text { meals } \\
& \text { Healthy eating } \\
& \text { motivation }
\end{aligned}
$$

motivation

$0,037 \quad 0,00311,8900,000$
Log-likelihood -1429.75
Pseudo $\mathrm{R}^{2}=0.135$
$\mathrm{~N}=1868$

$$
\begin{array}{ll}
0,377 & 0,1322,860 \\
0,708 & 0,1424,980
\end{array}
$$

0,004

$0,194 \quad 0,142 \quad 1,370$

0,172

$0,218 \quad 0,1341,630 \quad 0,103$

$0,482 \quad 0,1303,700$

0,000

$0,000 \quad 0,369 \quad 0,1592,320 \quad 0,020$

$0,484 \quad 0,1632,960 \quad 0,003$

$0,675 \quad 0,1564,340$

0,000

* Effects of socio-economic factors (age, gender, household, occupational status, education, social class, income) are not displayed.

Skipping a main meal has a statistically significant effect on dietary quality in all countries, but there is some variation in which meals matter where. In Denmark skipped lunches erode dietary quality, in Finland breakfast and lunch, in Norway lunch and dinner, and in Sweden lunch and 
dinner. In all four countries the duration of meals has no significant effect on dietary quality. Watching TV influences dietary quality in Denmark and Sweden, where those with a high proportion of TVmeals have lower dietary quality than those with a low proportion. Eating meals alone affects dietary quality in neither Finland nor Sweden, while in Denmark eating a very high proportion of meals alone results in dietary quality lower than that associated with eating a low or very low proportion of meals alone. In Norway eating a very high proportion of meals alone influences dietary quality negatively compared with eating a low proportion of meals alone. Finally, the number of eating events during the day predicts dietary quality significantly in all four countries. In each country, eating five or more meals per day influences dietary quality positively relative to the consumption of only 0-3 meals. Eating four meals per day also has a positive effect on dietary quality in Sweden and Denmark, as compared with eating 0-3 meals per day.

Table 10.3. exhibits two country-specific profiles with negative and positive effects on dietary quality. The profiles were defined on the basis of the eating activity variables that were statistically significant in the regression model. To illustrate, in Denmark the negative profile implies eating 03 meals during the day and skipping lunch, while the positive profile implies eating 5 or more meals per day, having lunch, and eating a low proportion of meals alone. Across all four countries the profiles amount to around $10-20 \%$ of the population, and they therefore represent quite prevalent daily eating activities.

Table 10.3. Country-specific profiles of daily eating activities with negative and positive effects on dietary quality

Activities with negative $\% \quad$ Activities with positive $\%$

effect

effect

(n)

(n)

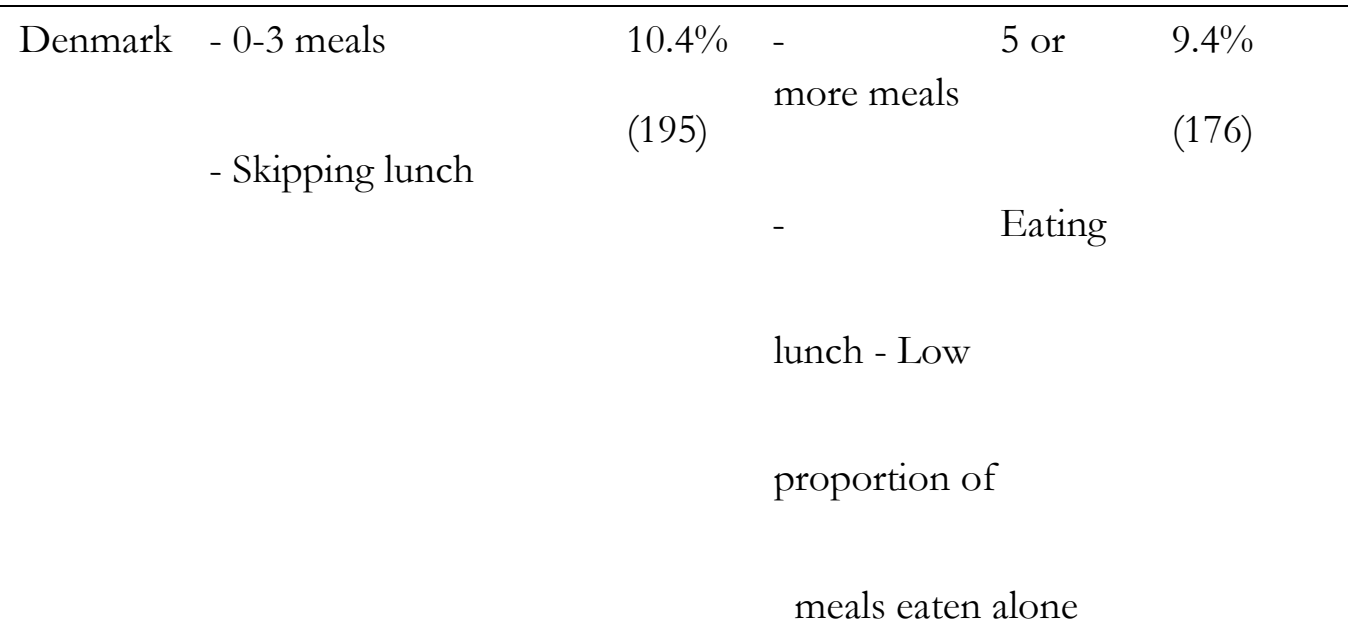




\begin{tabular}{|c|c|c|c|c|}
\hline Finland & - 0-3 meals & $10.9 \%$ & & $21.7 \%$ \\
\hline & - Skipping breakfast & (200) & - 5 or more meals & (398) \\
\hline & & & - Eating breakfast & \\
\hline & & & - Eating lunch & \\
\hline Norway & & $6.9 \%$ & & $20.2 \%$ \\
\hline & & (131) & -5 or more meals & (386) \\
\hline & $0-3$ meals & & & \\
\hline & & & - Eating dinner & \\
\hline & Eating & & & \\
\hline & alone (all meals) & & & \\
\hline Sweden & - $0-3$ meals & $10.8 \%$ & -5 or more meals & $16.2 \%$ \\
\hline & - Skipping dinner & (208) & - Eating dinner & (312) \\
\hline
\end{tabular}

Table 10.4. shows that healthy eating motivation influences dietary quality to quite a high degree. In Denmark the predicted probability of an unhealthy diet increases from 0.067 to 0.281 when moving from the $90^{\text {th }}$ to the $10^{\text {th }}$ percentile of motivation. This is more than a fourfold increase $(0.281 / 0.067=4.19)$. In the remaining countries, when comparing the $90^{\text {th }}$ percentile with the $10^{\text {th }}$ percentile of healthy eating motivation, the predicted probability of eating an unhealthy diet increases by $3.34(0.344 / 0.103)$ in Finland, 3.00 (0.231/0.077) in Norway, and $3.80(0.380 / .0100)$ in Sweden. 
Table 10.4. Predicted probabilities of dietary quality caused by eating activities during the day, country-specific profiles, and healthy eating motivation ${ }^{4}$

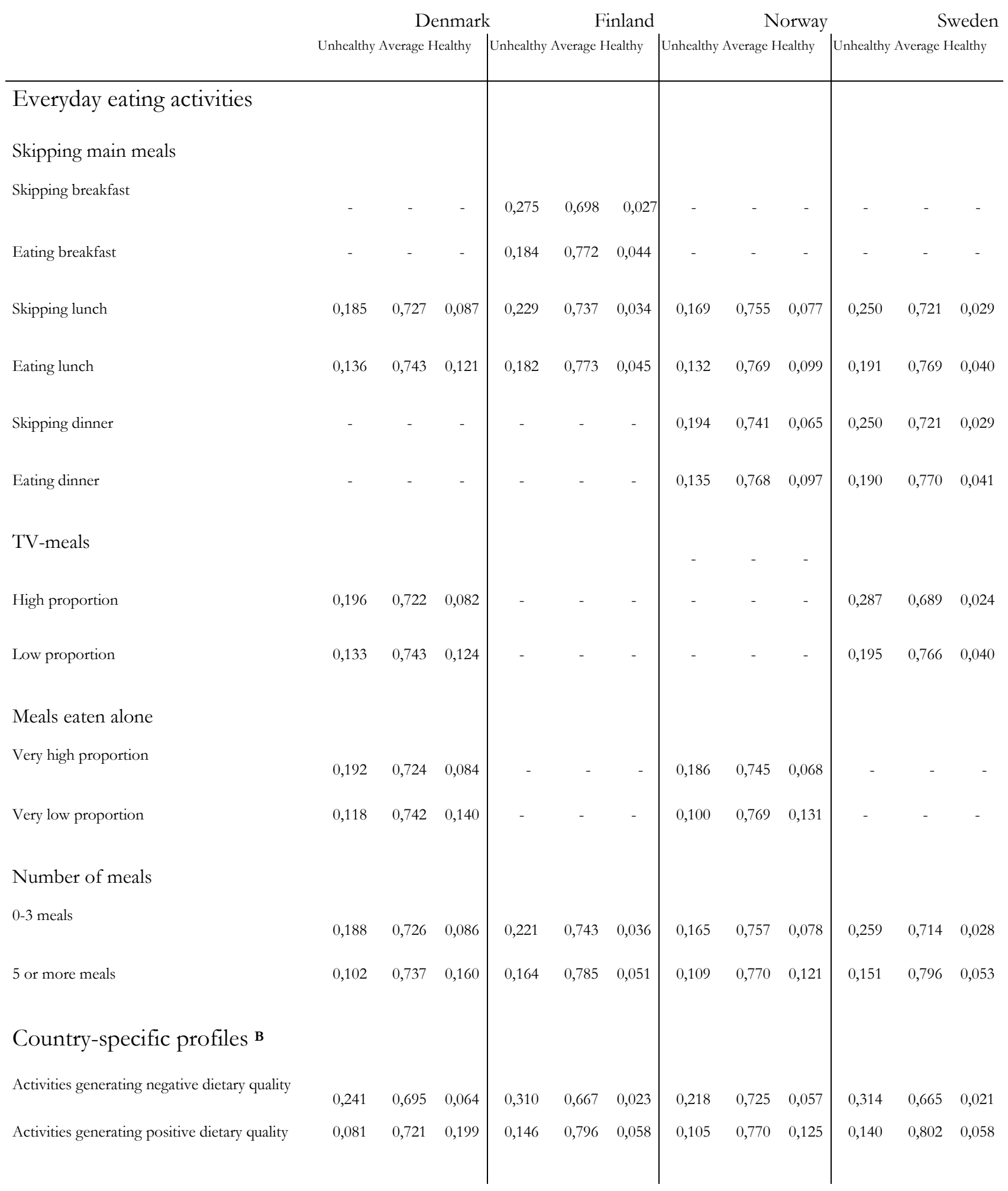

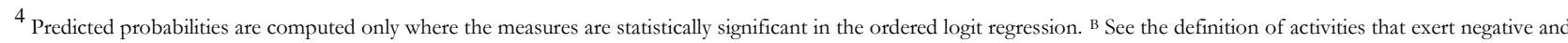
positive influence on dietary quality in Table 3
} 
Healthy eating motivation

Low motivation (10th percentile)

Median motivation (50th percentile)

High motivation (90th percentile)

\begin{tabular}{|c|c|c|c|c|c|c|c|c|c|c|c|}
\hline 0,281 & 0,666 & 0,053 & 0,344 & 0,637 & 0,020 & 0,231 & 0,716 & 0,053 & 0,380 & 0,604 & 0,016 \\
\hline 0,144 & 0,742 & 0,114 & 0,197 & 0,762 & 0,041 & 0,140 & 0,766 & 0,093 & 0,195 & 0,765 & 0,039 \\
\hline 0,067 & 0,702 & 0,231 & 0,103 & 0,813 & 0,084 & 0,077 & 0,755 & 0,168 & 0,100 & 0,818 & 0,082 \\
\hline
\end{tabular}

When they are compared with healthy eating motivation, the influence of the single measures of eating-related activities appear less decisive, as the changes in predicted probabilities are somewhat smaller. For instance, in Denmark the largest difference is observed in number of meals eaten per day, where eating 0-3 meals increases the predicted probability of unhealthy eating to 0.188 as compared with a 0.102 probability if five or more meals are eaten.

However, when we look at the country-specific positive and negative profiles defined in Table 3 we find that the changes in predicted probability are rather large. In Denmark the negative profile increases the predicted probability of belonging to the unhealthy dietary group to from 0.081 to 0.241 , which is almost a threefold increase $(0.241 / 0.081=2.98)$. Similarly, the positive profile increases the predicted probability of belonging to the healthy group from 0.064 to 0.199 . In the remaining countries, when we move from the positive profile to the negative profile of daily eating activities the predicted probability for eating an unhealthy diet increases by $2.12(0.310 / 0.146)$ in Finland, 2.08 (0.218/0.105) in Norway, and $2.24(0.314 / 0.140)$ in Sweden.

\section{Discussion}

The data above on the association between eating activities and dietary health in four Nordic populations suggest that the activities do indeed have an effect on dietary health even when motivation to eat healthily and socio-demographic factors are controlled for. They also reveal both similarities and differences between the Nordic countries in respect of which activities affect dietary health. First, there are trends pointing in the same direction in all countries: eating five or more meals a day and not skipping main meals advance healthy eating in every country; and frequent eating in a hurry does not affect dietary quality. Second, in some respects there are substantial country differences: eating alone and while watching TV reduces dietary health in some countries but not in others, and with respect to skipping main meals there are country differences in terms of which particular skipped meal affects dietary health.

The results of this study, then, suggest that the assumption that specific eating activities have an adverse effect on dietary health is not universally valid. For instance, eating alone, which has been found to be associated with less healthy eating (Pliner \& Bell 2009), is not associated with dietary health in Finland and Sweden. TV-viewing, which a number of studies have found to affect dietary health negatively (Blass, Anderson, Kirkorian, Pempek, Price, \& Koleini 2006; Feldman, Eisenberg, Neumark-Sztainer, \& Story 2007;Fuglestad, Jeffery, \& Sherwood 2012;Granner \& 
Mburia-Mwalili 2010; Jeffery \& French 1998; Liang, Kuhle, \& Veugelers 2009; Sisson, Shay, Broyles, \& Leyva 2012), was found in our study to have an effect on dietary health only in Denmark and Sweden. These national differences suggest that the role of a number of eatingrelated activities depends on cultural context.

The finding that eating five or more meals a day increased dietary health in all four countries corroborates other studies(Holmbäck, Ericson, Gullberg, \& Wirfält 2010; Kerver, Yang, Obayashi, Bianchi, \& Song 2006) and highlights the important role of the daily organization of eating. It suggests that, contrary to common belief, snacks are not always bad for health .( cf.Zizza \& Xu 2012). This is probably because they alleviate gnawing hunger pangs, and because if they accord with nutritional recommendations they can add healthy components into daily eating. Further analyses are needed, however, to examine the extent to which the content of snacks influences dietary health (cf.(Nicklas et al. 2014).

It is worth emphasizing that the effects of these eating practices are by no means trivial. Separately, the activities may not influence dietary health as decisively as motivation to eat healthily. But combinations of eating activities can have quite strong an effect. Thus country-specific negative eating profiles that are relatively common, encompassing 5-10\% of the populations examined, substantially increased the risk of having a poorer dietary quality.

Weaknesses of this study include the questionnaire's low completion rates and the fact that only one day of eating was recorded. Longer-term recording would have provided more valid measures of individual variation in daily practices and permitted finer-tuned analysis. But still, our data clearly offer relevant results on the ways in which the practices and dietary health are associated. It is true that the measure of dietary health employed here was originally developed to capture eating habits in Denmark (Toft, Kristoffersen, Ladelund, Bysted, Jakobsen, Lau, Jorgensen, BorchJohnsen, \& Ovesen 2007), implying that it may be less precise as an indicator of dietary quality in the other three countries. However, we believe that the potential measurement error resulting from this is outweighed by the benefits of being able to make international comparisons. It is a strength of the analysis that it is based on a large sample of adult populations from four countries, and that sociodemographic factors are controlled for. While more studies are needed to confirm our results, and to examine in more detail the role of food culture in the way eating activities relate to dietary health, our findings strongly suggest that, in public health promotion, more attention should be paid to practical and contextual aspects of the way people organize their eating. This shift is likely to make a valuable contribution to the development of nutrition policies and institutional measures that can support people's everyday efforts to eat more healthily. In particular, efforts to promote healthy eating should not focus exclusively on cognitive factors such as motivation and knowledge: the everyday contexts and locations of eating which facilitate or hamper healthy practices have a crucial role to play as well. 